\title{
Natural xanthone compounds as promising drug candidates against COVID-19 - An integrated molecular docking and dynamics simulation study
}

\section{Shravan Kumar Gunda}

1Bioinformatics Division, Prof. G. Ram Reddy Centre for Distance Education, Osmania University Hima Kumari $\mathbf{P}$

Centre for Biotechnology, Institute of Science \& Technology, Jawaharlal Nehru Technological University

Anuj Kumar

4Advance Bioinformatics Laboratory, Uttarakhand Council for Biotechnology (UCB)

\section{P B. Kavi Kishor}

5Department of Biotechnology, Vignan's Foundation for Science, Technology \& Research

Anil Kumar S ( $\nabla$ anilkumarou@gmail.com )

Department of Biotechnology, Vignan's Foundation for Science, Technology \& Research

\section{Research Article}

Keywords: Antiviral compounds, Covid19, Main protease, Molecular docking, Molecular simulations, Xanthones

Posted Date: February 10th, 2021

DOI: https://doi.org/10.21203/rs.3.rs-102884/v2

License: (c) (1) This work is licensed under a Creative Commons Attribution 4.0 International License. Read Full License 


\section{Abstract}

Severe acute respiratory syndrome coronavirus 2 (SARS-CoV-2) causes coronavirus disease 2019 (COVID-19). SARS-CoV-2 is known for its high pathogenicity and transmission due to the presence of polybasic cleavage sites. No specific drug is available for the treatment. To identify the potential inhibitors, we have performed molecular docking against the SARS-CoV-2 main protease (6Y84) with fifteen important natural xanthone compounds. The docking results showed all the compounds exhibited good binding energies and interactions with the main protease. The validation of representative docking complexes through molecular dynamics simulations showed that xanthones binds with a higher binding affinity and lower free energy than the standard ligand with Brasixanthone $\mathrm{C}$ and Brasixanthone $\mathrm{B}$ on $50 \mathrm{~ns}$. Natural xanthone compounds have also passed the Absorption, Distribution, Metabolism, and Excretion (ADME) property criteria as well as Lipinski's rule of five. The present integrated molecular docking and dynamics simulations study unveil the use of xanthones as potential antiviral agents against SARS-CoV-2.

\section{Introduction}

Coronaviruses cause respiratory tract infections in humans, ranging from a mild common cold to lethal diseases like SARS, MERS, and COVID-19. Severe acute respiratory syndrome coronavirus 2 (SARS-CoV2) causes coronavirus disease 2019 (COVID-19). It is also known as the 2019 novel coronavirus (2019nCoV) (Gorbalenya et al., 2020). The 2019-nCoV genome is 79\% similar to SARS-CoV (Lu et al., 2020a). It is a positive-sense, single-stranded RNA virus. As of 2 nd October 2020, around $34.5 \mathrm{M}$ people tested positive and 1.2M deaths were reported (https://www.worldometers.info/coronavirus/). COVID-19 pandemic was first recognized in December 2019 in Wuhan, China (Lu et al., 2020b), and spread in 215 countries and territories within a short period (https://www.worldometers.info/coronavirus/). The symptoms include fever, cold, cough, and respiratory syndrome (Chen et al., 2020). It spreads from human to human via respiratory droplets produced from coughs or sneezes. It enters humans by binding to the receptor angiotensin-converting enzyme 2 (ACE2) (Letko et al., 2020). High pathogenicity and transmission of SARS-CoV-2 are due to the presence of polybasic cleavage sites (Walls et al., 2020).

The recent development in technology has enabled researchers to understand the genetic, molecular, structural, and functional properties of 2019-nCoV to find appropriate targets against viruses. Several antiviral drugs like chloroquine, arbidol, remdesivir, and favipiravir are under consideration for treating 
COVID-19 (Dong et al., 2020). The development of medicine is the need of the hour. Xanthones are polyphenolic secondary metabolites with antiviral, anti-obesity, anti-HIV, antidiabetic, and anticancer activities (Anantachoke et al., 2012; Kumar et al., 2018). Hence xanthones are gaining importance as dietary substances (El-Seedi et al., 2020). Xanthones and their derivatives are isolated from different sources like plants, lichens, ferns, and fungi (Singh et al., 2016). The present study reports the identification of efficient xanthone compounds against SARS-CoV-2 main protease (6Y84) using integrated molecular docking and simulation studies.

\section{Materials And Methods}

\section{Ligand selection}

Fifteen natural xanthone compounds were selected as ligands from the PubChem database (Table 1) in SDF format (Kim et al., 2019).

\section{Molecular docking studies}

Molecular docking was performed to find the binding efficacy of protein and ligands using the AutoDock4.2 program (Morris et al., 1998). SARS-CoV-2 main protease (6Y84) protein was used in the present study. All the downloaded molecules were minimized by adding Gasteiger-Huckel charges and saved it into.mol2 format (Gasteiger and Marsili, 1980). Initially, the protein was loaded and deleted the water molecules and its ligands. The protein was imported and structurally optimized by adding all hydrogens and Kollman charges (Weiner et al., 1984). During docking, all the torsions were set to allow the rotation of the molecule. In the present molecular docking study, the protein is set as rigid and the ligand as flexible. Individually, all the xanthone molecules were docked. After adding the hydrogens, the model was saved in the PDBQT format. Thereafter, the selected ligands were individually prepared by optimizing the torsion angles and saved in the PDBQT format. PDBSUM was used for the selection of protein active sites (Laskowski et al., 2018). A grid was generated to identify the $X, Y, Z$ coordinates around the binding site of the protein individually. The grid dimensions were set to $60 \times 60 \times 60 \AA$ with points separated by $0.375 \AA$. The energy evaluations 250000 were selected, used 50 docking runs, and the population in the genetic algorithm. Ten final confirmations were generated for docking. Autodock uses a Lamarckian genetic algorithm (LGA) to search for the best conformation concerning the minimum binding energy $(\mathrm{kcal} / \mathrm{mol}$ ) and hydrogen bond distance between the protein and the ligand (Rajitha et al., 2017). For docking, default parameters are used.

\section{Drug likeness properties}

ADME calculations play a significant role in drug designing. All the fifteen ligand molecules analyzed for the likelihood of success, based on Lipinski's rule of five using Veber's rule (Veber et al., 2002) and Egan's rule. The polar surface area (TPSA) was calculated using the SWISSADME tool. 


\section{Molecular dynamics (MD) simulation}

MD simulation was performed on 50ns to evaluate the molecular docking results and to get a better understanding of the dynamics of representative docking complexes. Linux-based GROMOS96 43a1 force field embedded in the GROMACS 5.1 package was utilized for the determination of the thermodynamic stability of the docking complexes (Berendsen et al., 1995). For the MD simulation analysis, we followed the methods employed by Gajula et al. (2016), Jee et al. (2018), and Kumar et al. $(2018,2020)$. Using the PRODRG server, topology files of ligand molecules were prepared (Schüttelkopf and van Aalten, 2004). Dodecahedron box with simple point charge (SPC) waters was used to solvate the protein complexes. To neutralize the charge, $\mathrm{Na}^{+}$was added to the system as an opposing ion. Energy optimization was accomplished via 50,000 iteration steps using a steepest decent algorithm with a cutoff of up to $1000 \mathrm{kJmol}^{-1}$ to reduce the steric clashes. Before a production run, the constant number of particles, volume, and temperature (NVT) simulation was performed at $300 \mathrm{~K}$ set by Nose-Hoover thermostat and 1.01325 bar pressure to relax the protein-ligand complex. Finally, the production step, the run was carried for $50 \mathrm{~ns}$, and the trajectory was recorded at every $20 \mathrm{ps}$ time. After successful completion of MD simulation trajectories was analyzed using XMGRACE. The g RMS tool was used to calculate the Root mean square deviation (RMSD) variation in the protein backbone. While g RMSF tool was used to measure the overall Root mean square fluctuation (RMSF) in the atomic positions of protein $\mathrm{C}$ backbone. During the MD simulation, a rough measure of the compactness factor of the proteins was estimated using g gyrate tool embedded in GROMACS. The total solvent accessible surface area (SASA) was calculated using Gmxsasa tool. A 3.5 A distance cut-off was set to compute the distribution of intermolecular hydrogen bond length using $\mathrm{g} h$ bond.

\section{MM-GBSA binding free energy calculations}

Molecular mechanism generalized Born surface area (MM-GBSA) was implemented to get the binding free energy of the molecular interaction between docking complexes (Aldeghi et al., 2017). This employs ensembles obtained from simulation. Different components of binding free energy of the docking complexes were calculated using gmmpbsa module of GROMACS platform.

In the MM-GBSA calculation, the binding free energy for a docking complex was calculated using the below equation.

$M M / G B S A \Delta G_{\text {bind }}=G_{\text {complex }}-G_{\text {receptor }}-G_{\text {ligand }}$

where, $G_{\text {complex, }} G_{\text {receptor, }}$ and $G_{\text {ligand }}$ represent the free energies of the docking complex, receptor and ligand, respectively.

\section{Results And Discussion}




\section{Molecular docking interactions of natural xanthone compounds against SARS-CoV-2 main protease}

Molecular docking studies were performed for the prediction of the binding affinities of protein and ligands. Autodock is the most efficient method to predict potential-ligand interactions. AutoDock 4.2 uses a free binding energy assessment to assign the best binding conformations. Table 1 shows the docking results of 15 natural xanthone compounds. All the selected compounds for docking have some collective structural features. The comparison of binding energies against SARS-CoV-2 main protease (6Y84) protein reveals that the maximum compounds showed interactions with the main protease. Almost all molecules exhibited good binding energy values, indicating more thermodynamically favored interaction. Figure 1 shows the interactions with 6Y84.

The molecular docking interactions between different xanthone compounds and the main protease showed excellent binding energies. The compound Brasixanthone $C$ exhibited the highest binding energy of $-9.09 \mathrm{kcal} / \mathrm{mol}$ with the main protease. Nine compounds showed binding energies greater than 8.00 $\mathrm{kcal} / \mathrm{mol}$. Docking results demonstrated that Brasixanthone $\mathrm{C}$ made hydrogen bond interactions with His163, Thr26 amino acid residues of the main protease of COVID-19. Figures 1-3 represent proteinligand (3D and 2D).

Nine compounds showed binding energy more than $-8.00 \mathrm{kcal} / \mathrm{mol}$, four compounds exhibited binding energy more than $-7.00 \mathrm{kcal} / \mathrm{mol}$. The order of binding energies with $6 \mathrm{Y} 84$ are Brasixanthone $\mathrm{C}>$ Brasixanthone $\mathrm{B}>$ Caledonixanthone $\mathrm{C}>$ Globulixanthone $>$ Nigrolineaxanthone $\mathrm{N}>$ Shamixanthone $>$ Brasixanthone $\mathrm{D}>$ Caledonixanthone $\mathrm{B}>$ Teysmannic acid $>$ Caledonixanthone $\mathrm{F}>$ Varixanthone $>$ Globulixanthone $\mathrm{A}>$ Caledonixanthone $\mathrm{E}>$ Caledonixanthone $>$ Globulixanthone $\mathrm{C}$ respectively. It indicates that all the molecules showed excellent binding energies. The selected 15 natural compounds showed good binding energies and good interactions with SARS-CoV-2 main protease.

\section{Drug likeliness of the xanthones}

Brasixanthone B, Brasixanthone C, Brasixanthone D, Caledonixanthone B, Caledonixanthone C, Caledonixanthone D, Caledonixanthone E, Caledonixanthone F, Globulixanthone A, Globulixanthone B, Globulixanthone C, Nigrolineaxanthone N, Shamixanthone, Varixanthone, Teysmannic acid have the molecular weights $(378.42,410.42,424.45,278.31,296.32$, 342.35, 340.33, 358.35, 324.33, 378..42, $326.30,398.45,406.48,468.50,326.35) \mathrm{g} / \mathrm{ml}$ respectively. According to Lipinski's rule, all 15 molecules have a molecular weight $<500 \mathrm{~g} / \mathrm{mol}$ (Table 2). We also calculated the TPSA (topological polar surface area) for all the molecules. The topological polar surface area values for these compounds include 79.90, 109.36, 112.66, 39.45, 59.67, 100.13, 89.14, 109.36, 79.90, 79.90, 100.13, 111.12, 79.90, 126.43, $76.74 \AA^{2}$ respectively. According to the rule, the lowest topological polar surface area (TPSA) values always produce better results. Therefore, we noted that Caledonixanthone B produced a lower TPSA value of $39.45 \AA^{2}$, whereas Varixanthone produced a $126.43 \AA^{2}$ TPSA value. Selected molecules have much better 
behaved than the co-crystallized ligand. The (LogP) values of these compounds found to be 4.37, 3.67, 3.28, 3.60, 2.93, 3.20, 2.93, 2.31, 3.56, 4.44, 2.67, 3.90, 4.62, 3.25, 3.32 respectively. Predicted Consensus Log Po/w values suggest the easy absorption of the three molecules in the body. The three molecules can be easily synthesized.

\section{MD simulation}

MD simulation is an important method to study the biological macromolecules structure-to-function relationship, a crucial step for the drug discovery process (Gajula et al., 2016). In this present study, we performed MD simulation analysis on 50 ns for all the selected molecular docking complexes (Shamixanthone, Brasixanthone $B$, and Brasixanthone $\mathrm{C}$ ) to investigate their stability in the active site of the main protease. MD simulation results were analyzed from the predicted equilibrium trajectories by using a combination of a set of protocols including RMSD, RMSF, Rg, SASA, and interaction analysis of hydrogen bond and their stability during the MD simulation on 50 ns.

The RMSD plot analysis is crucial to provide information about the overall stability of the docking complex in terms of calculating the deviation in the initial molecular structure. As shown in Figure 4A, all three systems found significantly stable with variable deviations. The complex of the main protease with the Shamixanthone compound (black) depicted a stable and constant range of RMSD between 0.25 to $0.45 \mathrm{~nm}$, and similar results showed by Brasixanthone B natural compound (red). However, slight changes showed by Brasixanthone $C$ (green) on 0.25 to $0.50 \mathrm{~nm}$ during the initiation of the MD simulation on 50ns. The difference of calculated backbone RMSD values with Shamixanthone, Brasixanthone $B$, and Brasixanthone $C$ demonstrated that the main protease has conformational changes with Brasixanthone $C$ (green) at the initial stage. No significant changes in the complex with Shamixanthone, Brasixanthone B compounds during the whole MD simulation analysis.

As depicted in Figure 4B, the Brasixanthone C (green) showed the fluctuations at two different intervals on the whole simulation may have changed the confirmation in the pocket area of the main protease. The first stable confirmation between 1 to $9 \mathrm{~ns}$ on $0.3 \mathrm{~nm}$ and the second confirmation between $10 \mathrm{~ns}$ to 50 ns on $0.8 \mathrm{~nm}$ noticed respectively. The large fluctuation observed in the Brasixanthone $\mathrm{C}$ compound does not affect the overall structure of the protein structure. Shamixanthone (black), Brasixanthone B (red) showed the stability of 0.25 to $0.55 \mathrm{~nm}$. The Shamixanthone (black) showed the fluctuations from $30 \mathrm{~ns}$ to 50 ns between 0.3 to $0.55 \mathrm{~nm}$. During the entire MD simulation, these types of changes also did not affect the fluctuation of the protein backbone which depicted that the pocket area of the structure has the same fluctuation may be due to the large binding region and the presence of a loop. To understand the residue-wise fluctuations in the docking complexes, the RMSF plot of all the selected docking complexes was generated from the stable trajectory. As shown in Figure 4C, the RMSF plot suggested a similar trend of residue fluctuation for the main protease and its complex with all three compounds (Shamixanthone, Brasixanthone $B$, and Brasixanthone $C$ ). The RMSF plot analysis of selected docking complexes revealed that binding of all three compounds (Shamixanthone, Brasixanthone $B$, and Brasixanthone $C$ ) to the main 
protease was stable. Further, no significant changes were noticed on the flexibility of the whole MD simulation on $50 \mathrm{~ns}$.

\section{$\mathrm{Rg}$ and SASA analysis}

During MD simulation, radius and gyration is an essential indicator of compactness, stability, and folding of proteins structure. We have generated the Rg plot based on the intrinsic dynamics of all three selected docking complexes (Figure 5B). As evident from Figure 5B, docking complexes of the main protease with all the three compounds (Shamixanthone, Brasixanthone B, and Brasixanthone $\mathrm{C}$ ) have a range of average $\mathrm{Rg}$ of -2.1 to $2.2 \mathrm{~nm}$. The generated Rg plot of the main protease and its complexes with Shamixanthone, Brasixanthone $B$, and Brasixanthone $C$ compounds support condensed structure architecture as well as size. The outcomes of $\mathrm{Rg}$ analysis depict that all three natural compounds (Shamixanthone, Brasixanthone B, and Brasixanthone $\mathrm{C}$ ) have formed compact and stable complexes with the main protease of COVID-19. To validate the applicability of natural xanthone compounds as promising drug candidates against COVID-19, we have calculated the SASA for docking complexes of the main protease with Shamixanthone, Brasixanthone B, and Brasixanthone $\mathrm{C}$ molecules. During the MD simulation, SASA measures the area of receptor exposure to the solvents. As shown in Figure 5A, the calculated SASA value exhibited between $130-150 \mathrm{~nm}^{2}$ justified that the binding of selected xanthone compounds does not affect the folding of the main protease very much. SASA analysis concludes that selected docking complexes impressively stable after the binding of Shamixanthone, Brasixanthone B, and Brasixanthone $\mathrm{C}$ compounds to the pocket area of the main protease.

\section{Hydrogen bonds analysis}

In a docking complex, hydrogen bonding plays a critical role in the identification of the binding strength of a ligand molecule with the receptor protein. Natural xanthone compounds Shamixanthone (black), Brasixanthone $B$ (red), and Brasixanthone $\mathrm{C}$ (green) have a constant range of intermolecular hydrogen bonding with the main protease with the range of 0 to 12 in throughout the MD simulation on $50 \mathrm{~ns}$. Figures $5 C$ and $D$ represent hydrogen bond number and their distribution. Hydrogen bond analysis not only confirms the docking results but also validates the non-existence of any types of conformational change in binding sites during MD simulation. Based on the hydrogen bond analysis, we can conclude that the complexes of selected xanthone derivatives with the main protease of COVID-19 are stable throughout the MD simulation process.

\section{Free binding energy analysis}

The free binding energy was calculated for all three systems (Shamixanthone, Brasixanthone B, and Brasixanthone $\mathrm{C}$ ). The MM/GBSA free energy is a well-known method to estimate the affinities of the ligand-binding in a docking complex. As suggested by previous reports, 100-200 snapshots are enough 
to assess the binding free energies (Kumar et al., 2020). The calculated binding energy for selected three docking complexes provided in Table 3. The Vanderwaal energy component compared with every complex, the Brasixanthone B $(4399.778+/-96.831 \mathrm{~kJ} / \mathrm{mol})$ has less effect on binding affinity while Shamixanthone $(4832.073+/-96.433 \mathrm{~kJ} / \mathrm{mol})$ and Brasixanthone $\mathrm{C}(4856.322+/-101.790 \mathrm{~kJ} / \mathrm{mol})$ have a strong binding affinity with the main protease. As evident from Table 2, Shamixanthone (4872.208 +/$96.811 \mathrm{~kJ} / \mathrm{mol})$ and Brasixanthone $\mathrm{C}(4888.760+/-102.053 \mathrm{~kJ} / \mathrm{mol})$ demonstrated a similar effect of binding energy while moderate in Brasixanthone $B(4456.305+/-98.17 \mathrm{~kJ} / \mathrm{mol})$. Free binding energy calculations revealed that all these three xanthone derivatives could consequently prove to be a potential target against the main protease if COVID-19.

\section{Conclusion}

The present work, molecular docking, and molecular dynamics simulations studies were analyzed to explore the binding mechanism of natural xanthone compounds to the COVID19 main protease protein, to enable the design of natural compound derivatives as novel coronavirus inhibitors. The binding confirmation of selected natural compounds and their binding energies was calculated by the molecular docking method and simulation studies. The binding energies of the natural xanthone compounds to the main protease proteins have an excellent correlation. The docking results for all the compounds provide insight into the structural requirement for the biological activity. The binding mode of the highest-scoring compounds will be useful in designing novel inhibitors against COVID-19. However, further in vitro and in vivo evaluations of these xanthone compounds are necessary to investigate and gain more insight into COVID-19 for drug discovery.

\section{Acknowledgements}

SAK acknowledges the SERB-NPDF fellowship (PDF/2015/000929).

\section{Conflict of interest}

The authors declare that the research was conducted in the absence of any commercial or financial relationships that could be construed as a potential conflict of interest.

\section{References}

Aldeghi, M., Bodkin, M. J., Knapp, S., and Biggin, P. C. (2017). Statistical analysis on the performance of molecular mechanics poisson boltzmann surface area versus absolute binding free energy calculations: Bromodomains as a case study. J. Chem. Inf. Model. 57, 2203-2221. doi: 10.1021/acs.jcim.7b00347

Anantachoke, N., Tuchinda, P., Kuhakarn, C., Pohmakotr, M., and Reutrakul, V. (2012). Prenylated caged xanthones: chemistry and biology. Pharm. Biol. 50, 78-91. doi: 10.3109/13880209.2011.636176 
Berendsen, H. J. C., van der Spoel, D., and van Drunen, R. (1995). GROMACS: A message-passing parallel molecular dynamics implementation. Comput. Phys. Commun. 91, 43-56. doi: 10.1016/00104655(95)00042-e

Chen, N., Zhou, M., Dong, X., Qu, J., Gong, F., Han, Y., Qiu, Y., Wang, J., Liu, Y., Wei, Y., Xia, J., Yu, T., Zhang, $X$. , and Zhang, L. (2020). Epidemiological and clinical characteristics of 99 cases of 2019 novel coronavirus pneumonia in Wuhan, China: A descriptive study. Lancet. 395, 507-513. doi: $10.1016 /$ S0140-6736(20)30211-7

Dong, L., Hu, S., and Gao, J. (2020). Discovering drugs to treat coronavirus disease 2019 (COVID-19). Drug Discov. Ther. 14, 58-60. doi: 10.5582/ddt.2020.01012.

El-Seedi, H. R., Salem, M. A., Khattab, O. M., El-Wahed, A. A., El-Kersh, D. M., Khalifa, S. A.M., et al. (2020). "Dietary xanthones" in Handbook of dietary phytochemicals, eds. J. Xiao, S. D. Sarker, and Y. Asakawa, (Springer Nature Singapore Pte Ltd, Singapore), 1-22.

Gajula, M. N. P., Kumar, A., and ljaq, J. (2016). Protocol for molecular dynamics simulations of proteins. Bio-Protocol. 6, 1-11. doi: 10.21769/BioProtoc.2051

Gasteiger, J., and Marsili, M. (1980). Iterative partial equalization of orbital electronegativity-a rapid access to atomic charges. Tetrahedron 36, 3219-3228. doi: 10.1016/0040-4020(80)80168-2

Gorbalenya, A. E., Baker, S. C., Baric, R. S., de Groot, R. J., Drosten, C., Gulyaeva, A. A., et al. (2020). The species Severe acute respiratory syndrome-related coronavirus: classifying 2019-nCoV and naming it SARS-CoV-2. Nat. Microbiol. 5, 536-544. doi: 10.1038/s41564-020-0695-Z

Jee, B., Kumar, S., Yadav, R., Singh, Y., Kumar, A., and Sharma, M. (2018). Ursolic acid and carvacrol may be potential inhibitors of dormancy protein small heat shock protein16.3 of Mycobacterium tuberculosis. J. Biomol. Struct. Dyn. 36, 3434-3443.

Kim, S., Chen, J., Cheng, T., Gindulyte, A., He, J., He, S., et al. (2019). PubChem 2019 update: improved access to chemical data. Nucleic Acids Res. 47, D1102-D1109. doi: 10.1093/nar/gky1033

Kumar, A., Choudhir, G., Shukla, S. K., Sharma, M., Tyagi, P., Bhushan, A., and Rathore, M. (2020). Identification of phytochemical inhibitors against main protease of COVID-19 using molecular modeling approaches. J. Biomol. Struct. Dyn. doi: 10.1080/07391102.2020.1772112

Kumar, A., Kumar, R., Sharma, M., Kumar, U., Gajula, M. N. P., and Singh, K. P. (2018). Uttarakhand medicinal plants database (UMPDB): A platform for exploring genomic, chemical, and traditional knowledge. Data. 3, 7. doi: doi.org/10.3390/data3010007

Laskowski, R. A., Jabłońska, J., Pravda, L., Vařeková, R. S., and Thornton, J. M. (2018). PDBsum: Structural summaries of PDB entries. Protein Sci. 27, 129-134. doi: 10.1002/pro.3289 
Letko,M., Marzi, A., and Munster, V. (2020). Functional assessment of cell entry and receptor usage for SARS-CoV-2 and other lineage B betacoronaviruses. Nat. Microbiol. 5, 562-569. doi: 10.1038/s41564020-0688-y

Lu, H., Stratton, C. W., and Tang, Y. W. (2020b). Outbreak of pneumonia of unknown etiology in Wuhan, China: The mystery and the miracle. J. Med. Virol. 92, 401-402. doi: 10.1002/jmv.25678

Lu, R., Zhao, X., Li, J., Niu, P., Yang, B., Wu, H., Wang, W., Song, H., Huang, B., Zhu, N., and Bi, Y. (2020a). Genomic characterisation and epidemiology of 2019 novel coronavirus: implications for virus origins and receptor binding. Lancet. 395, 565-574. doi: https://doi.org/10.1016/s0140-6736(20)30251-8

Morris, G. M., Goodsell, D. S., Halliday, R. S., Huey, R., Hart W. E., Belew, R. K., et al. (1998). Automated docking using a Lamarckian generic algorithm and empirical binding free energy function. J. Comput. Chem. 19, 1639-1662.

Rajitha, B., Saleha, B., Rajashaker, B., Reddy, G. A., Lingaiah, N., Sirisha, K., et al. (2017). Potential antimicrobial agents from triazole-functionalized $2 \mathrm{H}$-benzo[b] [1,4]oxazin-3(4H)-ones. Bioorg. Med. Chem. Lett. 27, 5158-5162. doi: 10.1016/j.bmcl.2017.10.061

Schüttelkopf, A. W., and van Aalten, D. M. F. (2004). PRODRG: a tool for high-throughput crystallography of protein-ligand complexes. Acta Crystallogr. D Biol. Crystallogr. 60, 1355-1363.

Singh, A., Kaur, N., Sharma, S., and Bedi, P.M. S. (2016). Recent progress in biologically active xanthones. J. Chem. Pharm. Res. 8, 75-131.

Veber, D. F., Johnson, S. F., Hung-Yuan Cheng, H. Y., Smith, B. R., Ward, K. W., and Kopple, K. D. (2002). Molecular properties that influence the oral bioavailability of drug candidates. J. Med. Chem. 45, 26152623. doi: $10.1021 / \mathrm{jm} 020017 \mathrm{n}$

Walls, A. C., Park, Y. J., Tortorici, M. A., Wall, A., McGuire, A. T., and Veesler, D. (2020). Structure, function and antigenicity of the SARS-CoV-2 spike glycoprotein. Cell 181, 281-292.e6. doi:

10.1016/j.cell.2020.02.058

Weiner, S. J., Kollman, P. A., Case, D. A., Singh, U. C., Ghio, C., Alagona, G., et al. (1984). A new force field for molecular mechanical simulation of nucleic acids and proteins. J. Am. Chem. Soc. 106, 765-784.

\section{Tables}

Due to technical limitations, the tables have been placed in a supplemental file.

\section{Figures}




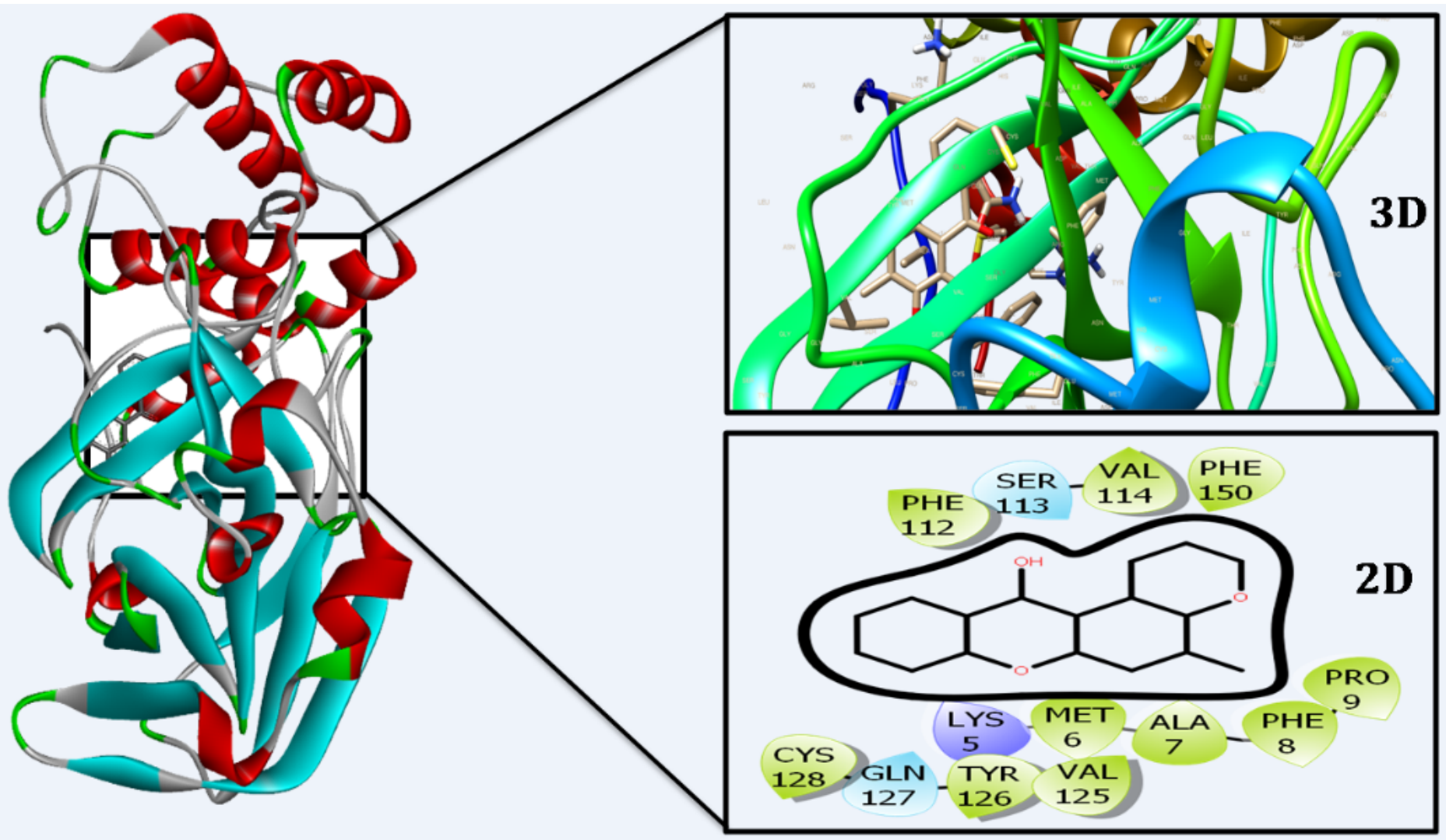

Figure 1

Protein-ligand interaction diagram (3D and 2D) of complex between main protease and Shamixanthone

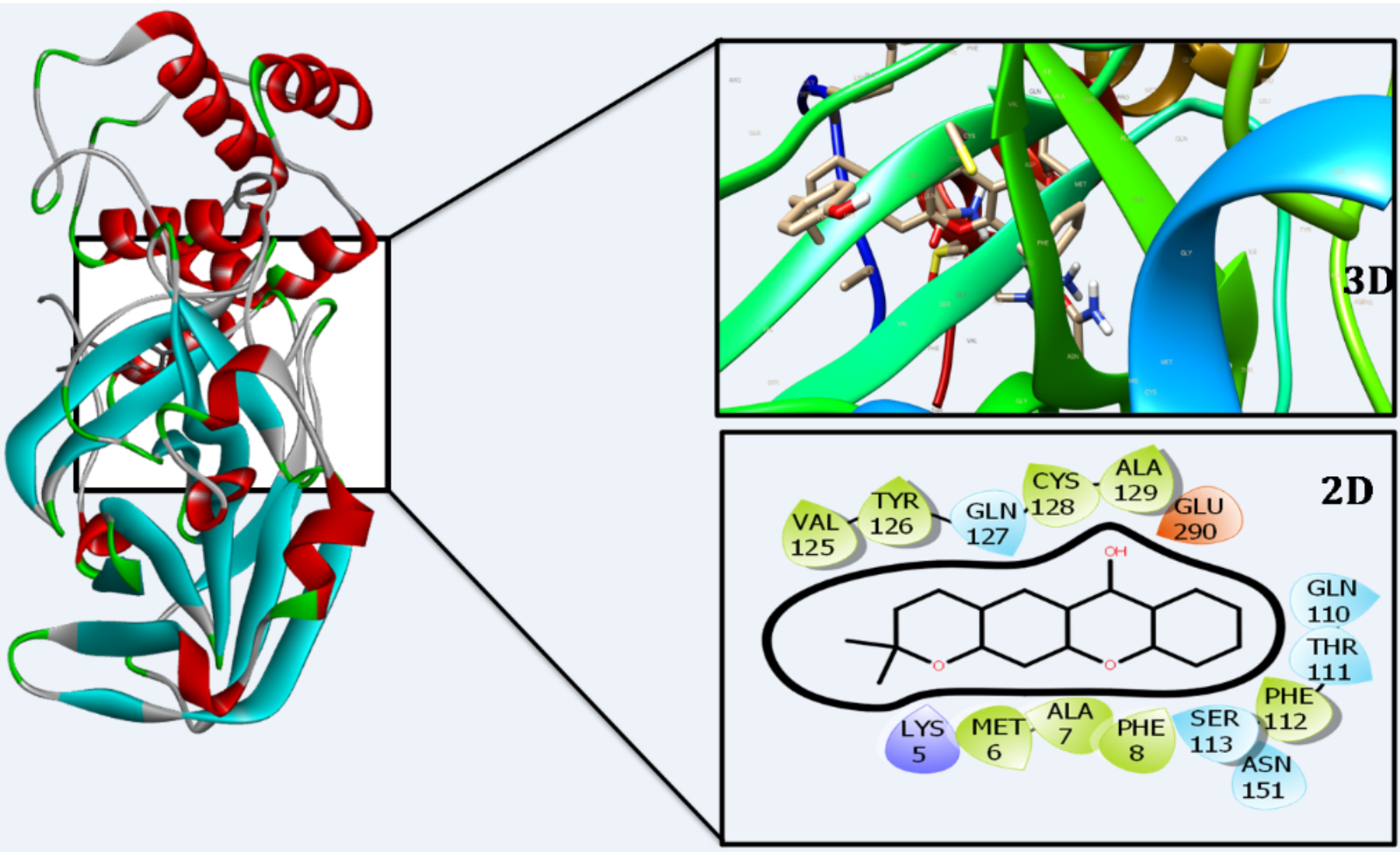


Figure 2

Protein-ligand interaction diagram (3D and 2D) of complex between main protease and Brasixanthone B

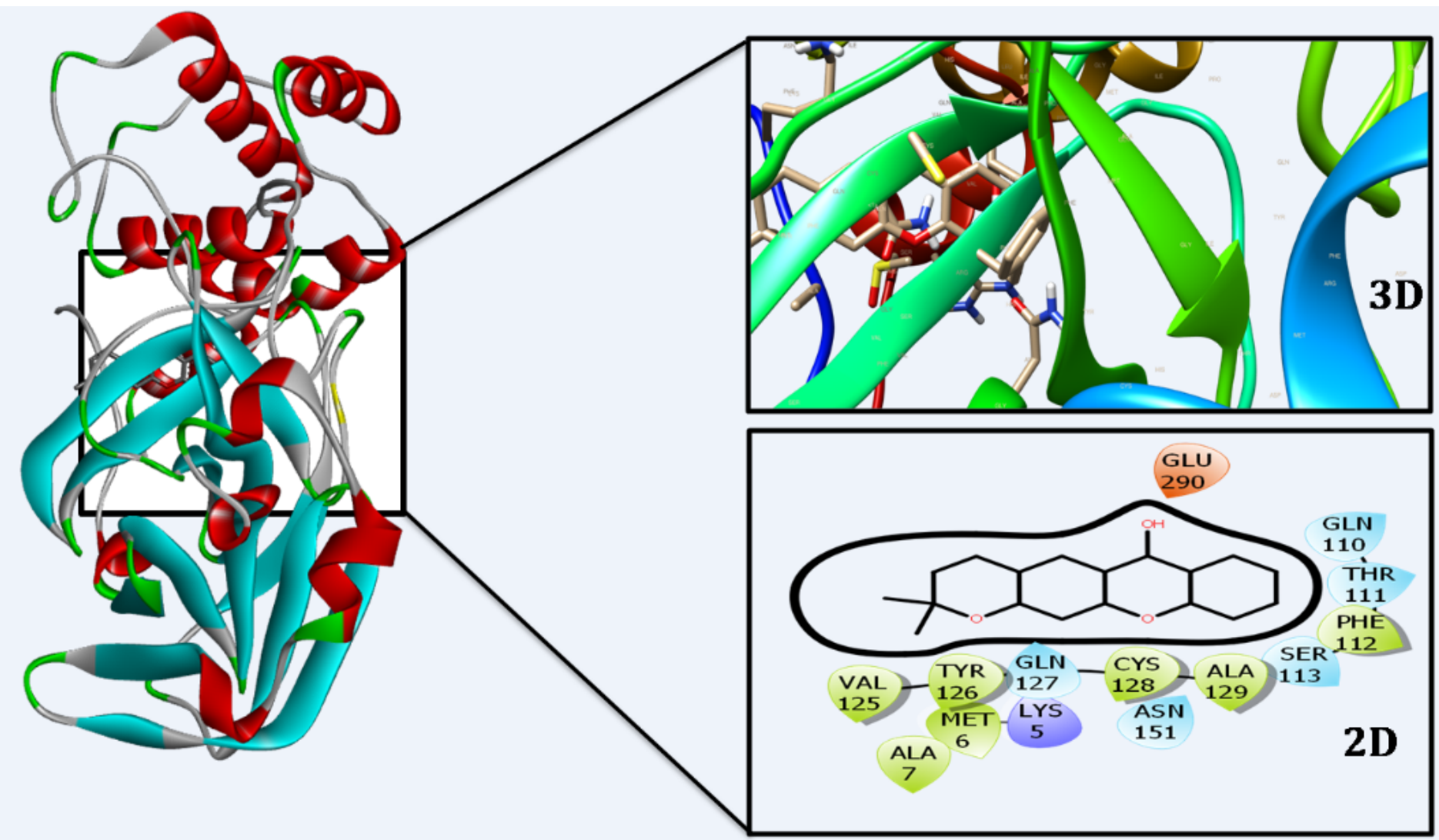

Figure 3

Protein-ligand interaction diagram (3D and 2D) of complex between main protease and Brasixanthone $\mathrm{C}$ 

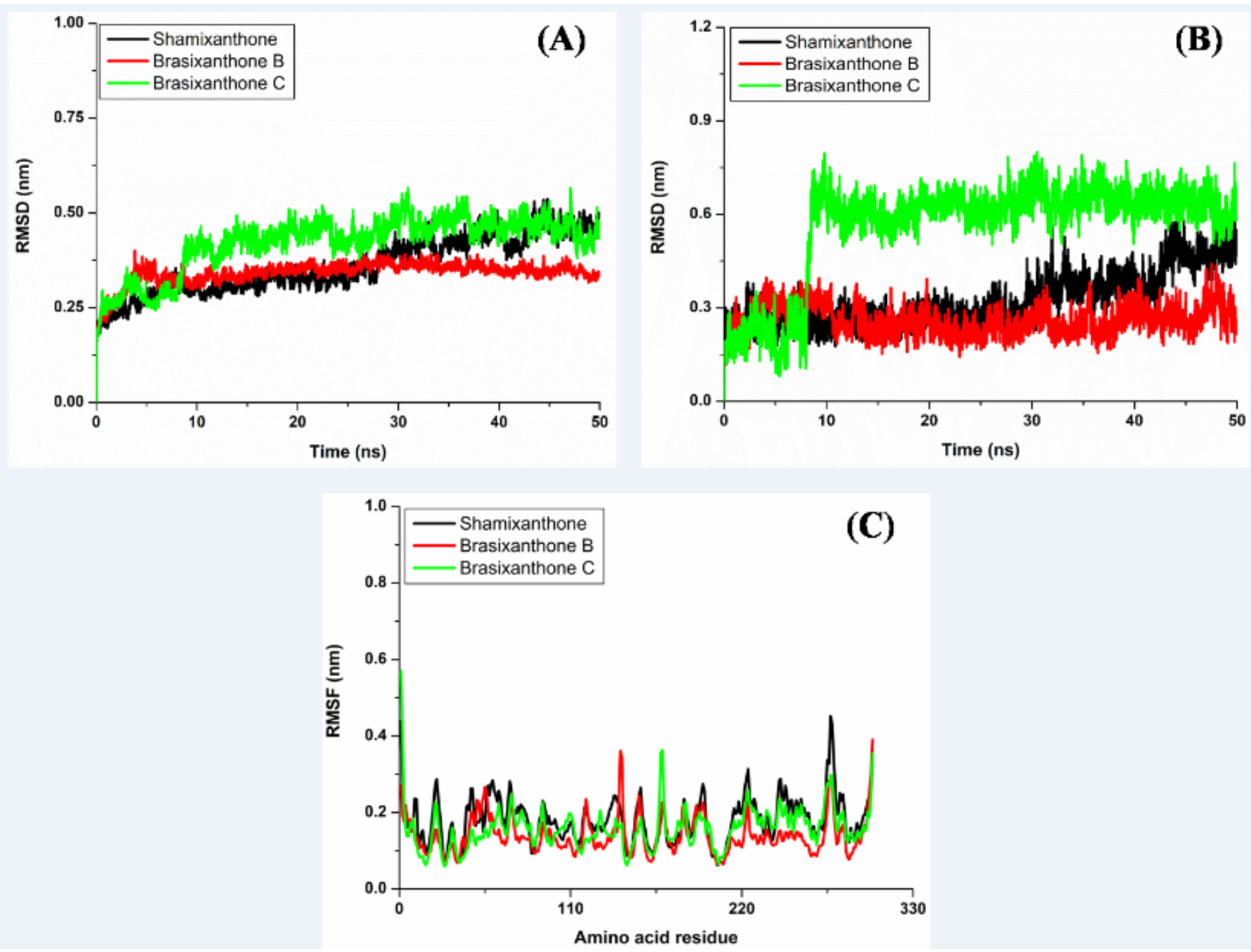

\section{Figure 4}

RMSD and RMSF analysis of main protease and ligands i.e. Shamixanthone (black), Brasixanthone B (red) and Brasixanthone C (green): (A) RMSD plot of docking complexes; (B) RMSD of protein backbone structure; (C) Analysis of RMSF of Ca during MD simulation on 50 ns. 

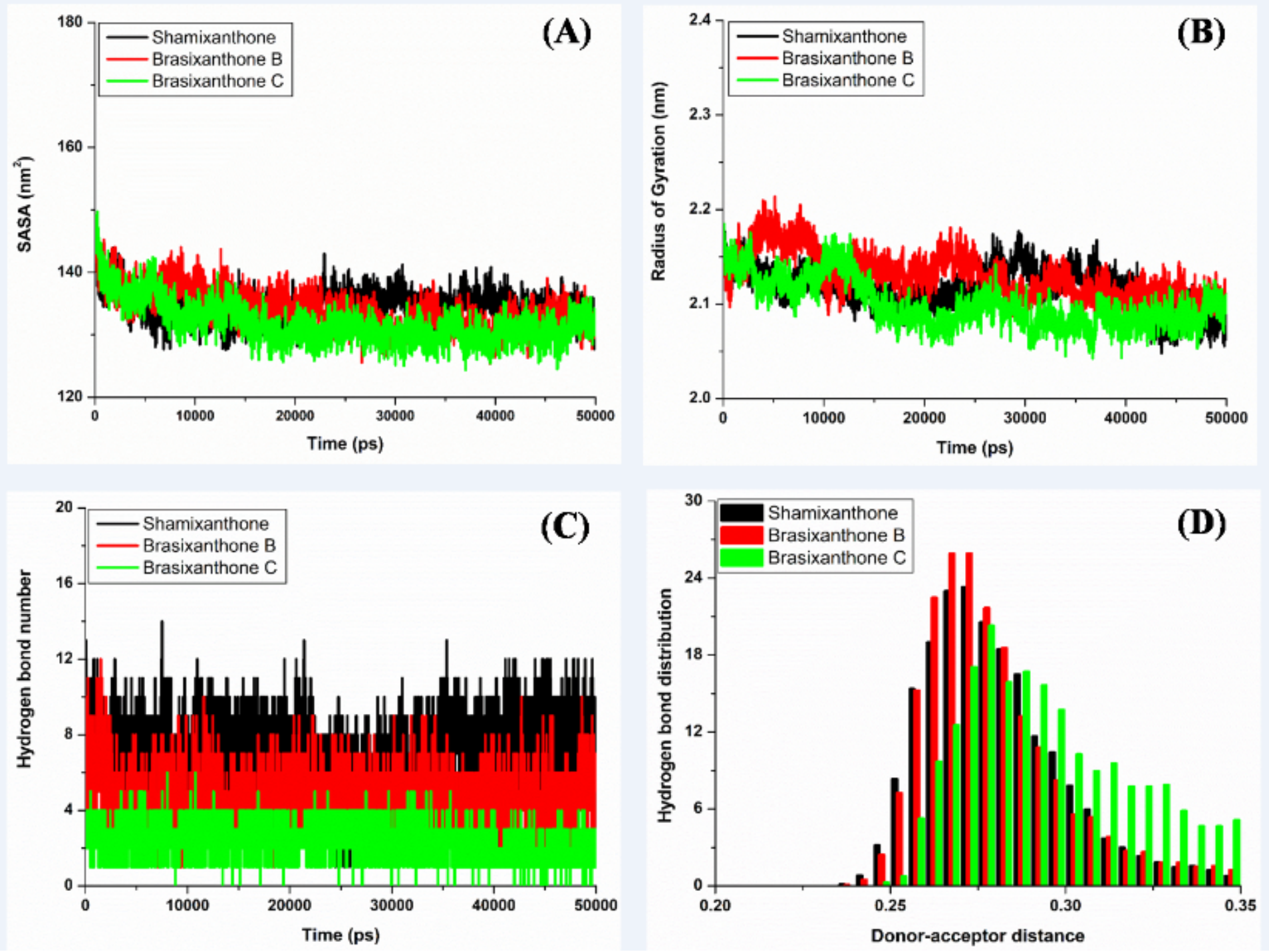

Figure 5

SASA, Rg, hydrogen bond number and distribution analysis of main protease docking complexes with Shamixanthone (black), Brasixanthone B (red) and Brasixanthone C (green): (A) SASA plot; (B) Rg plot analysis; (C) Hydrogen bond number analysis; (D) Hydrogen bond distribution analysis plot.

\section{Supplementary Files}

This is a list of supplementary files associated with this preprint. Click to download.

- GundaetalTables.pdf 